Here we present a unique case report of development of mixed glandular-neuroendocrine cancer four years after complete resection of advanced adenocarcinoma of the gallbladder in a 72-year-old woman. Primary treatment consisted of extended cholecystectomy with wedge resection of the gallbladder bed including more than $2 \mathrm{~cm}$ of normal liver and dissection of the regional lymph nodes. Recurrent tumour was treated by extended right hemihepatectomy and adjuvant chemotherapy.

This case is the first description of mixed glandular-neuroendocrine cancer of the gallbladder and represents the long-term follow-up of a surviving patient with this stage of the disease. The authors discuss possible explanations of such a phenomenon and emphasize the need for evaluation of neuroendocrine differentiation in gallbladder carcinomas, which can be misdiagnosed, as it may be related to better prognosis, and may indicate the necessity of adjuvant chemotherapy application.

Key words: gallbladder, mixed tumour, neuroendocrine carcinoma, adenocarcinomas.

\section{Mixed glandular-neuroendocrine cancer of the gallbladder. Report of a case}

\author{
Adam Durczyński ${ }^{1}$, Dariusz Szymański ${ }^{1}$, Stanisław Sporny ${ }^{2}$, \\ Janusz Strzelczyk ${ }^{1}$
}

1Department of General and Transplant Surgery, Medical University of Lodz, Norbert Barlicki Memorial Teaching Hospital, Lodz, Poland

2Department of Dental Pathology, Medical University of Lodz, The Clinical-Didactic Centre Lodz, Poland

\section{Introduction}

Presence of both exocrine and endocrine cells in adenocarcinomas of the gallbladder has been well recognized [1]. However, mixed glandularneuroendocrine cell carcinomas of the gallbladder, which according to the WHO classification (2000) belong to neuroendocrine tumours (NET), are very rare [2]. So far, only a few cases of these gastrointestinal tumours have been described worldwide, and most of them were referred to as adenocarcinoma with carcinoid component. Composite adenocarcinoma with a non-secretory, highly malignant variant of neuroendocrine tumours such as neuroendocrine cell carcinoma is even more uncommon.

The influence of neuroendocrine differentiation in carcinomas of the gallbladder on prognosis and survival of patients is still unknown. According to previous studies, mixed cancers of the extrahepatic biliary tract may be associated with much poorer prognosis than that of other gallbladder carcinomas, irrespective of stage of the disease [3]. Earlier investigations also revealed that the behaviour of mixed glandular-neuroendocrine gastrointestinal tumours is determined mainly by the adenocarcinomatous element, regardless of the presence of endocrine cells [4]. In contrast, recent clinicopathological studies indicate that glandular/neuroendocrine malignant tumours of the gastrointestinal tract have a better prognosis than common carcinoma [5]. However, due to the small number of reported cases, the results of all these studies are still not representative. Nevertheless, from a clinical point of view, morphological subtyping of composite exocrine-endocrine cancer of the gallbladder seems to have fundamental implications for the selection of treatment modality, because anticancer activity of subsequent chemotherapy regimens in these tumours has been proven, in contrast to pure adenocarcinoma of the gallbladder, which is resistant to cytostatic treatment [6]. Thus, because of the wider range of novel treatment modalities, the reasonable conclusion is that for patients with mixed exocrine-endocrine tumours life expectancy may be prolonged. Nevertheless, the prognosis of malignant gallbladder tumours still remains very poor, and when they are diagnosed at a late stage, the tumours are usually fatal.

To our knowledge, this is the first case report of recurrence of mixed glandular-neuroendocrine cancer following macroscopically complete resection of advanced adenocarcinoma of the gallbladder. This case is the first description of coincidence of these tumours and represents one of the longest follow-ups of a surviving patient with this stage of the disease. Additionally, the authors discuss possible histogenetic and technical explanations of such a coincidence. Moreover, the authors emphasize the need for evaluation of neuroendocrine differentiation in gallbladder carcinomas, which can be misdiagnosed, as it may be related to better prognosis, and may indicate the necessity of adjuvant chemotherapy application. 


\section{Case presentation}

A 72-year-old woman was admitted to the hospital on April 2004 with the presumptive diagnosis of cholecystolithiasis. The patient was qualified to undergo laparoscopic cholecystectomy. Intraoperatively, after initiation of pneumoperitoneum and insertion of four trocars through the abdominal wall, the presence of a tumour in the fundus of the gallbladder infiltrating neighbouring hepatic tissue was confirmed. Other organs were intact macroscopically. The decision of conversion from laparoscopic to open surgery was made. Extended cholecystectomy with wedge resection of the gallbladder bed including more than $2 \mathrm{~cm}$ of normal liver and dissection of the regional lymph nodes were performed. Histological examination of the resected specimen revealed adenocarcinoma of the gallbladder $5 \times$ $\times 3 \mathrm{~cm}$ in size (fig. 1). The patient has been followed up without any adjuvant treatment and after 53 months recurrence was confirmed during computed tomography examination. CT scans revealed a $38 \times 34 \times 41 \mathrm{~mm}$ hypodense focal noncircumscribed, faintly enhanced lesion suspected to be a malignant neoplasm within the right lobe and hilum of the enlarged liver. Spleen, pancreas, kidneys and adrenal glands were without focal lesions. Additionally, abdominal

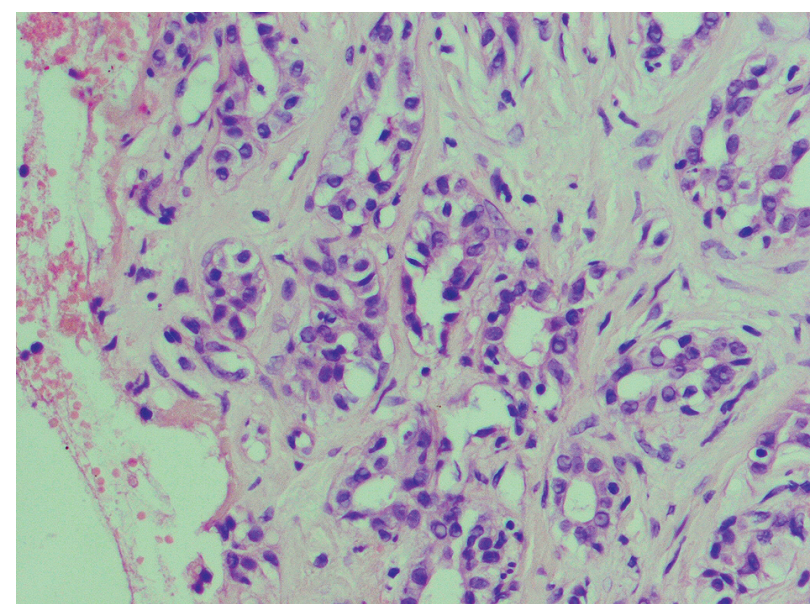

Fig. 1. Adenocarcinoma of the gallbladder, HE (300x)

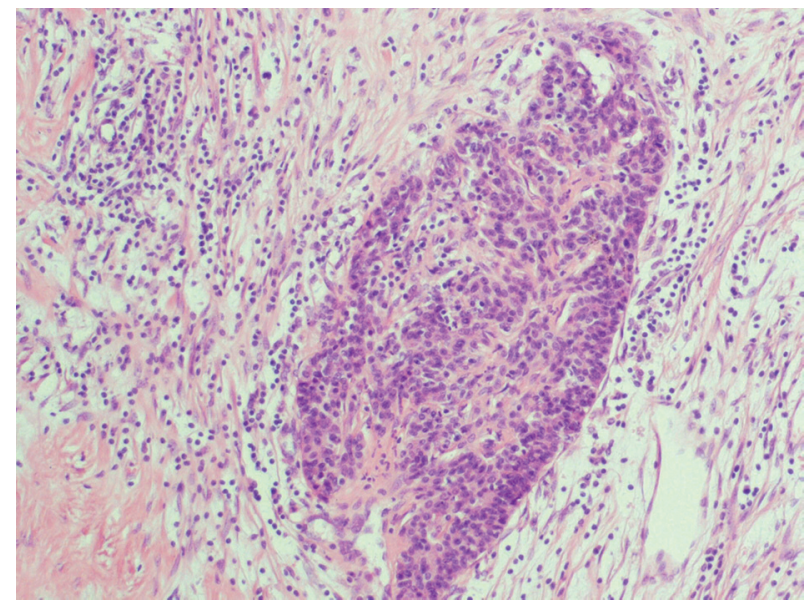

Fig. 3. Cuneiform nests of the neuroendocrine cancer, HE (200x) lymph nodes were not enlarged. The patient complained of right upper abdominal quadrant intermittent colic pain, nausea and epigastric fullness of one month duration. On physical examination no signs of somatic disease were found. On this basis, the patient was qualified for surgical treatment and underwent extended right hemihepatectomy. Postoperative recovery was not complicated.

Macroscopically part of the liver $15 \times 10 \times 7 \mathrm{~cm}$ in size with a solitary solid tumour of $4.5 \mathrm{~cm}$ in maximum diameter was confirmed. Routine haematoxylin/eosin stained sections from a formalin-fixed, paraffin-embedded surgical resection specimen were examined. Histologically, this tumour was composed of both adenocarcinoma and endocrine cell carcinoma (fig. 2). The two elements were randomly distributed, but also with apparent separation between them in many areas. The adenocarcinoma was a moderately differentiated tumour. In addition the endocrine carcinoma consisted of mainly cuneiform nests of medium-sized amphiphilic cells (fig. 3). The tumour was locally extended to the adjacent liver tissue (fig. 4), although no features of incision line infiltration were found. The Ki-67 index was assessed by avidin biotin conjugate (ABC) technique. The proliferation index evaluated by this method

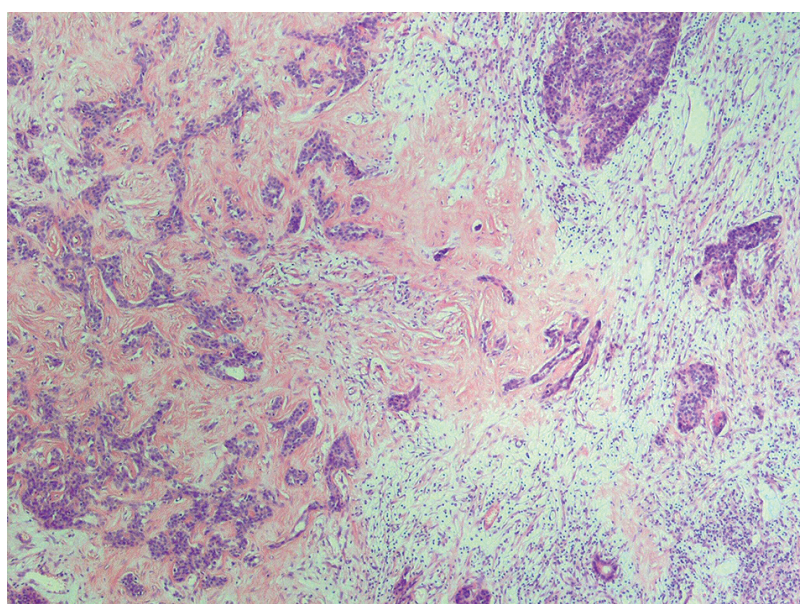

Fig. 2. Recurrent composite glandular-endocrine cell carcinoma of the gallbladder, $\mathrm{HE}(120 \times)$

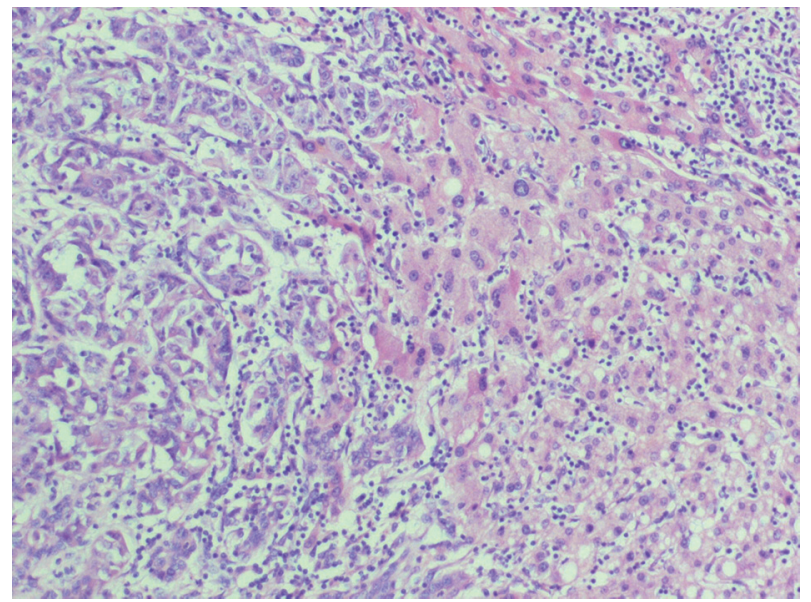

Fig. 4. Mixed tumour of the gallbladder infiltrating normal liver parenchyma, HE (300x) 


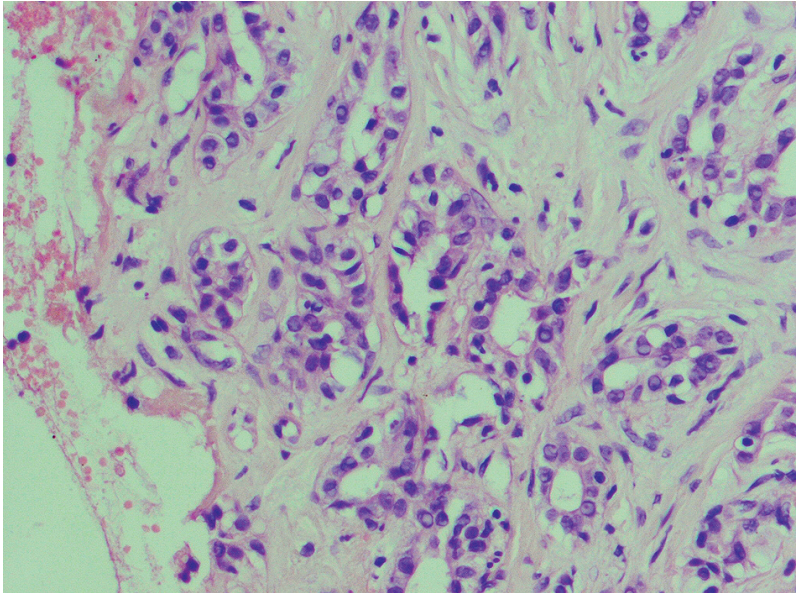

Fig. 5. Immunohistochemical staining for synaptophysin in the mixed cancer. ABC method (300x)

was 30\%. In further immunohistochemical staining synaptophysin was locally strongly positive (fig. 5) but chromogranin staining was negative. Thus, on the basis of these observations, the diagnosis of mixed glandular-neuroendocrine cancer of the gallbladder was established. The cell population contained approximately equal amounts of both tumour components.

\section{Discussion}

Because the natural history of malignant gallbladder tumours is greatly asymptomatic in early stages, they are not diagnosed until the cancer has spread and infiltrates adjacent organs, which is related to the poor survival rate. The overall survival is significantly prolonged in incidental diagnosis of early stage gallbladder cancer. Local recurrences following potentially curative resection of gallbladder cancer are most common, being the main pattern of treatment failure. The overall median postoperative time to disease recurrence is less than one year [7]. Most commonly locoregional recurrences of gallbladder cancer occur concomitantly with distant site metastases. Isolated local disease is rather rare. The prognosis among patients with recurrent gallbladder cancer is very poor and overall survival does not exceed 2 years. In our case the patient survived 4 years without any recurrence of the disease, which is one of the longest periods for this stage of the cancer. Moreover, histopathological examination of the recurrent tumour revealed a different histotype.

In consideration of such long patient survival, the reasonable question to ask is whether the primary resected gallbladder tumour was adenocarcinoma or mixed endocrine/exocrine cancer. The authors did not establish a definite diagnosis of composite tumour during repeated examination of primary gallbladder tissue with negative immunohistochemical staining of synaptophysin. Thus, the previous diagnosis of gallbladder carcinoma was confirmed. However, the final histopathological examination of these tumours may not be accurate for multiple reasons [8]. Neuroendocrine tumours are relatively rare neoplasms and therefore many histopathologists may not be experienced in their diagnosis. Furthermore, during routine histopathological investigation only part of the tumour is precisely examined. Thus, there is still a possibility that some percentage of mixed glandular/neuroendocrine carcinomas may be misdiagnosed as one of the invasive components of these tumours, which may hypothetically have taken place in the described case.

Unfortunately, establishment of a diagnosis of pure gallbladder adenocarcinoma in cases of mixed malignant tumours has not been implicated with any other treatment modalities, in contrast to neuroendocrine cell carcinoma, which is proved to be more susceptible to chemotherapy. Complete resection of the tumour with clear surgical margins remains the main possibility to achieve satisfactory survival in neuroendocrine cancers, although objective response rates to chemotherapy may reach $30-40 \%$, which indeed may be associated with survival prolongation, if it is applied in an adjuvant regimen. Moreover, chemotherapy may be applicable among patients with low-grade recurrent neuroendocrine cancers or primary dissemination.

Still, histopathology of mixed neuroendocrine tumours is not clear. On the basis of immunohistochemical stainings of gastrointestinal cancer cells it was proved that almost $40 \%$ of examined adenocarcinomas were positive for chromogranin and polypeptide hormones, which are characteristic for neuroendocrine tumours [9]. Some investigators suggest the possibility of coincidence of adenocarcinoma and neuroendocrine cancers in gastrointestinal malignancies [10-13]. In our case the two elements appear rather separated, indicating the presence of two independent tumours of different histotype.

Histogenesis of such tumours, as our case described, may be controversial. One of the possible histogenetic explanations of development of glandular/neuroendocrine carcinomas is synchronous or metachronous neoplastic differentiation of two different cell types [14-16]. So far this concept has been discouraged and the hypothesis of bidirectional neoplastic differentiation of multipotential stem cells has been promoted, because it is more likely to occur. However, in our patient the composite tumour developed secondary to the resection of primary pure adenocarcinoma of the gallbladder, which may be an argument in favour of the previous hypothesis. Indeed, such a concomitance has to be associated with accumulation of genetic alterations, which predispose to development of synchronous or metachronous double tumours, and further molecular studies are necessary to elucidate these controversies.

\section{References}

1. Hsu W, Deziel DJ, Gould VE, Warren WH, Gooch GT, Staren ED. Neuroendocrine differentiation and prognosis of extrahepatic biliary tract carcinomas. Surgery 1991; 110: 604-10.

2. Solcia E, Klöppel G, Sobin LH. Endocrine Tumors of the Gastrointestinal Tract. Histological Typing of Endocrine Tumors, 2nd edn. Geneva 2000; 61-68.

3. Yannakou N, Rizos S, Parissi-Mathiou P, Smailis D, Charanioti1 S, Dervenis C. Mixed (composite) glandular-endocrine cell carcinoma of the gallbladder. HPB 2001; 3: 7-9. 
4. Ahn JE, Byun JH, Ko MS, Park SH, Lee MG. Neuroendocrine carcinoma of the gallbladder causing hyperinsulinaemic hypoglycaemia. Clin Radiol 2007; 62: 391-4.

5. Grabowski P, Scherübl H. Expression of neuroendocrine markers in undifferentiated carcinomas of the gastrointestinal tract. J Clin Oncol 2005; 23: 4795-6.

6. Kos-Kudła B, Bolanowski M, Handkiewicz-Junak D, et al. Zalecenia diagnostyczno-lecznicze w guzach neuroendokrynnych układu pokarmowego (rekomendowane przez Polską Sieć guzów Neuroendokrynnych). Endokrynologia Polska 2008; 1: 41-56.

7. Jarnagin W, Leyo R, Little S, Klimstra D, D'Angelica M, Dematteo R, Wagman R, Blumgart L, Yuman F. Patterns of initial disease recurrence after resection of gallbladder carcinoma and hilar cholangiocarcinomalmplications for adjuvant therapeutic strategies. Cancer 2003; 98: 1689-700.

8. Hofler H, Stier A, Schusdziarra V i wsp. Classification of neuroendocrine tumors of the gastrointestinal tract and pancreas and its therapeutic relevance. Chirurg 1997; 68: 107-5.

9. Yao GY, Zhou JL, Lai MD, et al. Neuroendocrine markers in adenocarcinomas: an investigation of 356 cases. World J Gastroenterol 2003; 9: 858-61.

10. Fossmark R, Zhao CM, Martinsen TC, et al. Dedifferentiation of enterochromaffin-like cells in gastric cancer of hypergastrinemic cotton rats. Apmis 2005; 113: 436-9.

11. Sugihara A, Nakasho K, Yamada N, et al. Neuroendocrine differentiation of periodic-acid Schiff and Alcian blue-negative signet-ring cell-like cells and tubular adenocarcinoma cells within a gastric cancer. Scand J Gastroenterol 2004; 39: 795-800.

12. Syder AJ, Karam SM, Mills JC, et al. A transgenic mouse model of metastatic carcinoma involving transdifferentiation of a gastrin epithelial lineage progenitor to a neuroendocrine phenotype. Proc Natl Acad Sci U S A 2004; 101: 4471-6.

13. Habal N, Sims C, Bilchik AJ. Gastrointestinal carcinoid tumors and second primary malignancies. J Surg Oncol 2000; 75: 310-6.

14. Klappenbach RS, Kurman RJ, Sinclair CF, James LP. Composite carcinoma-carcinoid tumours of the gastrointestinal tract. A morphologic, histochemical and immunohistochemical study. Am J Clin Pathol 1985; 84: 137-43.

15. Lewin K. Carcinoid tumours and mixed (composite) glandularendocrine cell carcinomas. Am J Surg Pathol 1987; 11 (suppl 1): 71-86.

16. Wisniewski M, Toker C. Composite tumours of the gallbladder exhibiting both carcinomatous and carcinoid patterns. Am J Gastroenterol 1972; 58: 633-7.

\section{Address for correspondence}

\section{Adam Durczyński}

Department of General and Transplant Surgery

Medical University of Lodz

Norbert Barlicki Memorial Teaching Hospital

ul. Kopcińskiego 22

90-153 Lodz

e-mail: durek@retsat1.com.p 